\title{
Heterogeneity in local density allows a positive evolutionary relationship between self-fertilisation and dispersal
}

\author{
Running title: Evolution of selfing and dispersal \\ James Rodger ${ }^{\mathrm{a}, \mathrm{b}}$, Pietro Landi ${ }^{\mathrm{a}, \mathrm{c}^{*}}$, Cang Hui ${ }^{\mathrm{a}, \mathrm{d}}$ \\ ${ }^{a}$ Theoretical Ecology Group, Department of Mathematical Sciences, Stellenbosch University, \\ Matieland 7602, South Africa \\ bUniversity of Lausanne, Department of Ecology and Evolution, Biophore, 1015 Lausanne, \\ Switzerland \\ ${ }^{c}$ Evolution and Ecology Program, International Institute for Applied Systems Analysis, Schloßplatz 1, \\ 2361 Laxenburg, Austria \\ ${ }^{\mathrm{d}}$ Mathematical and Physical Biosciences, African Institute for Mathematical Sciences, Muizenberg \\ 7945 , South Africa \\ *corresponding author email address: landi@sun.ac.za
}

\section{Keywords}

Mating system

Adaptive dynamics

Self-fertilisation

Dispersal

Baker's Law

Allee effects

\section{Acknowledgements}

We thank Kalle Parvinen for discussion and Ophelie Ronce and two anonymous reviewers for their thorough and constructive comments which greatly improved the quality of this paper. This research was funded by the National Research Foundation (NRF) of South Africa (grants 89967, 109244 and 109683).

This article has been accepted for publication and undergone full peer review but has not been through the copyediting, typesetting, pagination and proofreading process, which may lead to differences between this version and the Version of Record. Please cite this article as doi: 10.1111/evo.13562.

This article is protected by copyright. All rights reserved. 


\title{
Author contributions
}

J.G.R., P.L. and C.H. conceived the study. P.L. built the model and investigated it, in consultation with J.G.R. and C.H. The paper was written by J.G.R., with editorial input from P.L. and C.H.

J.G.R. and P.L. contributed equally to the paper (Co-first authors).

\section{Data archiving}

There is no data associated with this paper.

\begin{abstract}
Despite empirical evidence for a positive relationship between dispersal and self-fertilisation (selfing), theoretical work predicts that these traits should always be negatively correlated, and the Good Coloniser Syndrome of high dispersal and selfing (Cf. Baker's Law) should not evolve. Critically, previous work assumes that adult density is spatiotemporally homogeneous, so selfing results in identical offspring production for all patches, eliminating the benefit of dispersal for escaping from local resource competition. We investigate the joint evolution of dispersal and selfing in a demographically structured metapopulation model where local density is spatiotemporally heterogeneous due to extinction-recolonisation dynamics. Selfing alleviates outcrossing failure due to low local density (an Allee Effect) while dispersal alleviates competition through dispersal of propagules from high- to lowdensity patches. Because local density is spatiotemporally heterogenous in our model, selfing does not eliminate heterogeneity in competition, so dispersal remains beneficial even under full selfing. Hence the Good Coloniser Syndrome is evolutionarily stable under a broad range of conditions, and both negative and positive relationships between dispersal and selfing are possible, depending on the environment. Our model thus accommodates positive empirical relationships between dispersal and selfing not predicted by previous theoretical work and provides additional explanations for negative relationships.
\end{abstract}




\section{Keywords}

Mating system; Adaptive dynamics; Self-fertilisation; Dispersal; Baker's Law; Allee effects

\section{Introduction}

As self-fertilisation (selfing) and dispersal respond to common selective pressures and influence selection on each other, they should evolve jointly (Massol and Cheptou 2009, Busch 2011, Massol and Cheptou 2011b, Duputié and Massol 2013). Both these traits affect gene flow and colonisation success, which in turn have strong effects on geographical distributions, biological invasions and speciation (Busch 2011, Massol and Cheptou 2011b, Hargreaves and Eckert 2014, Pannell 2015, Hui and Richardson, 2017). Thus, the joint evolution of selfing and dispersal and the consequent emergence of syndromes of mating and dispersal have broad implications (Cheptou and Massol 2009).

Baker's Law (Baker 1955, Stebbins 1957) states that species that can self-fertilise should be better colonisers because selfing assures reproduction when mate or pollinator availability limits outcross reproduction in the new environment. Based on this, it can be expected that highly dispersive, frequently colonising species should benefit from selfing, leading to evolution of the Good Coloniser Syndrome of high dispersal and high selfing (argument developed by Cheptou and Massol 2009). However, quantitative theoretical investigations accounting for the effect of selfing rate on dispersal rate evolution and vice versa predict only syndromes of high dispersal with no self-fertilisation (i.e. pure outcrossing) or high selfing with no dispersal but never the Good Coloniser Syndrome (Cheptou and Massol 2009, Massol and Cheptou 2011a, Sun and Cheptou 2012). This implies that the relationship 
between these traits should always be negative. Although Cheptou and Massol (2009) cite some limited empirical evidence in support of this (Price and Jain 1981, Renner and Ricklefs 1995, Sutherland 2004), their results do not accommodate other empirical studies showing positive relationships between selfing ability and dispersal ability across multiple species or populations within a species (Darling et al. 2008, De Waal et al. 2014). Thus, notwithstanding that theory focuses on rates and empirical work on abilities for selfing and dispersal (Duputié and Massol 2013, Pannell et al. 2015), there is a need to expand theory to accommodate positive as well as negative relationships between these two traits.

Selection on dispersal depends on the relative advantages of offspring staying versus leaving their site of origin (Clobert et al. 2004, Ronce 2007). In the metapopulation framework (Levins 1969, Hanski 1988), which has been widely used for theoretical studies of dispersal, dispersal rate can be defined as the proportion of propagules (the dispersing stage, e.g. a seed or larva) emigrating from its patch (Hastings 1983, Duputié and Massol 2013). In general, spatiotemporal heterogeneity selects positively on dispersal because the patches that are most favourable for production of propagules in one generation are not the most favourable ones for them to complete their life cycles and to reproduce in the next generation (Hastings 1983, Levin et al. 1984, McPeek and Holt 1992, Massol and Débarre 2015). For example, when stochastic local extinction causes spatiotemporal heterogeneity in density-dependent competition, dispersal can be favoured because it reduces competition (van Valen 1971, Comins 1980, Parvinen 2006). This benefit is usually referred to as "escape from competition", even though dispersal reduces competition for both dispersed and nondispersed propagules. Dispersal may also be favoured for avoidance of kin competition (Hamilton and May 1977) and inbreeding (Motro 1991, Perrin and Mazalov 1999). 
Negative selection on dispersal comes from costs, such as the risk of failing to reach destination habitat after leaving the habitat of origin (cost of dispersal: Hamilton and May 1977) as well as energetic costs of dispersal structures and movements (Ronce 2007). Dispersal is also selected against when patch conditions are only heterogeneous in space and do not vary in time, because most propagules are then produced in the better patches and benefit from staying there rather than dispersing (Hastings 1983). Thus, positive temporal autocorrelation generally selects against dispersal and negative autocorrelation in favour of it (Olivieri et al. 1995, Massol and Débarre 2015). In addition, where small or low-density populations suffer from mate limitation (i.e., under an Allee effect), individuals that reach vacant habitat may reproduce poorly if only small numbers of propagules arrive, selecting against dispersal (Robinet and Liebhold 2009). This last result comes from a study on gypsy moths, which are always unisexual and therefore cannot evolve selfing. However, in a system where selfing is possible, selection could favour increased selfing instead of reduced dispersal, potentially giving rise to the Good Coloniser Syndrome (Good Coloniser Syndrome).

While most studies of selfing focus on continuous populations (e.g., Lande and Schemske 1985, Charlesworth and Charlesworth 1987, Cheptou and Fenster 2004, Morgan and Wilson 2005), investigating the implications of colonisation for selfing evolution requires a spatially structured setting, such as a metapopulation. Three main factors govern selection on selfing: reproductive assurance, the transmission advantage and inbreeding depression (Lloyd 1992, Barrett 2010, Eckert et al. 2006, Karron et al. 2012). Reproductive assurance is the benefit of selfing in mitigating mate or pollinator limitation. In metapopulations, this can favour evolution of selfing when empty patches are colonised by small numbers of propagules (Pannell and Barrett 1998, Dornier et al. 2008), as mate limitation frequently occurs in small and sparse populations (Leimu et al., 2006, Gascoigne et al. 2009). This is an example of the 
Allee effect, defined generally as a reduction in performance due to low abundance (positively density-dependent performance, Stephens et al. 1999). Selfing is also promoted by the transmission advantage: that selfing variants can both self and outcross, and so pass on more copies of their genes to the next generation than strict outcrossers (Fisher 1941). Inbreeding depression, the poorer performance of offspring from inbreeding, is the main force opposing the evolution of selfing (Darwin 1876, Charlesworth and Charlesworth 1987, Husband and Schemske 1996). It arises mainly from increased expression of recessive deleterious alleles under inbreeding compared to outbreeding, although reduction in heterozygote advantage (due to overdominance) also plays a role (Wright 1977, Charlesworth and Charlesworth 1999, Charlesworth and Willis 2009). Selection can remove deleterious recessive alleles if self-fertilisation rates remain high for several generations, reducing inbreeding depression and providing a positive feedback in evolution of selfing (Lande and Schemske 1985, Barrett and Charlesworth 1991, Crnokrak and Barrett 2002, Lande and Porcher 2015).

Despite the rich theoretical literatures on the evolution of selfing and of dispersal individually (see reviews by Clobert et al. 2004, Ronce 2007, Barrett 2010, Karron et al. 2012), few models have considered the evolution of both traits simultaneously (Ravigné et al. 2006, Cheptou and Massol 2009). The previously studied model of Cheptou and Massol (2009) represents a metapopulation where presence versus absence of pollinators fluctuates in habitat patches but all patches have identical density of adult plants. Increasing the rate of pollinator failure (absence of pollinators) selects for selfing. However, when selfing rate is high, spatiotemporal heterogeneity in seed production, and consequently local resource competition, is low and the cost of dispersal outweighs the benefit. Due to effect of selfing rate on dispersal evolution, this model predicts that only syndromes of no dispersal and high selfing or dispersal and no selfing should be evolutionarily stable, precluding the Good 
Coloniser Syndrome (Cheptou and Massol 2009, Massol and Cheptou 2011a, Sun and Cheptou 2012). Although not particularly emphasised (e.g., Massol and Cheptou 2011a, Sun and Cheptou 2012, Auld and de Casas 2013), this model invokes a mechanism for selection which only applies to plants (stochastic pollinator fluctuations) and assumes a metapopulation in which local density is homogeneous in space and time.

In this study, we investigate the joint evolution of selfing and dispersal in a demographically structured metapopulation model, where spatiotemporal heterogeneity in density arises from stochastic local extinction, followed by recolonisation and population growth. Selfing is selected to assure reproduction under mate limitation, which affects both animals and plants. Previous investigations that have used the same general modelling framework, but with only one evolving trait considered, show that positive selection on dispersal arises from spatiotemporal heterogeneity in local density (Parvinen 2006), and positive selection on selfing arises from the presence of recently recolonised patches with low density (Dornier et al. 2008). These results suggest that a positive evolutionary relationship between selfing and dispersal may occur and the Good Coloniser Syndrome may occur be evolutionarily stable when dispersal and selfing evolve simultaneously under the same conditions (Busch 2011, Massol and Cheptou 2011b).

\section{Model and methods}

\subsection{Metapopulation model}

We adapted and extended the metapopulation models of Parvinen (2006) and Dornier et al. (2008) for evolution of dispersal and selfing respectively to allow evolution of both traits simultaneously. Our model considers evolution of a diploid hermaphrodite species with an annual life history in a metapopulation of an infinite number of identical patches occupied by local populations. Time is discrete and events in each time step (year) are as follows. 
- Adult individuals produce female gametes (ovules for plants or ova for animals), a fraction of which are self-fertilised (prior selfing, Lloyd 1992). Due to inbreeding depression, a fraction of self-fertilised ovules/ova fail to produce propagules (for example seeds, fertilised eggs or actively dispersing young).

- The ovules that are not self-fertilised are available for cross fertilisation. The fraction of these ovules that are actually cross-fertilised (and produces propagules) is positively related to local density (i.e., there is an Allee effect due to mate limitation) and it is assumed that cross-fertilisation only occurs between individuals within the same local population.

- Of all propagules produced, a fraction disperses (emigrates) and the remainder stays in the local population.

- A fraction of dispersed propagules is lost (the cost of dispersal), and successfully dispersed propagules are evenly distributed among all patches, following the (infinite) island model of dispersal (Wright, 1931).

- After emigration and immigration, carrying capacity of patches imposes competition (establishment from propagule to adult is thus negatively density dependent).

- Finally, a fraction of local populations suffer extinction due to environmental stochasticity. Individuals in the remaining populations go on to produce propagules in the following year.

Events thus happen each year in the following order: reproduction, emigration, immigration, competition, and local extinction. Assuming a very large number of patches, the metapopulation has an age distribution of local populations $p_{\tau}=e(1-e)^{\tau}$, where $e$ is local 
population extinction rate and $\tau$ the age of these populations, with $\tau=0$ for newly extinct populations (Ronce et al. 2000). In a local population, per capita reproduction $(\lambda)$ is described by

$$
\lambda(s, N)=f(s(1-\delta)+(1-s) A(N)),
$$

where fertility $f$ is the number of ovules produced per individual, selfing rate $s$ is the fraction of ovules that are self-fertilised, and $\delta$ is the fraction of self-fertilised ovules dying due to inbreeding depression (Cheptou 2004). Due to the Allee effect in mate limitation, the fraction $(1-s) A(N)$ ovules are cross fertilised, where $A(N)=N /(a+N)$ (Cheptou 2004), $N$ is the local population density of adults and parameter $a$ is the local population density at which half of the ovules/ova available for outcrossing are actually outcrossed. Here we refer consistently to local density, although as all habitat patches are assumed to be physically identical, local population density is equivalent to size, assuming no kin competition or mating.

The density of propagules in each local population following emigration and immigration is:

$$
S_{\tau}=(1-d) \lambda\left(s, N_{\tau}\right) N_{\tau}+(1-c) D(t),
$$

where dispersal rate $d$ is the fraction of propagules dispersing (emigrating), $c$ is the fraction of these lost during dispersal (cost of dispersal) and $D(t)$ is the dispersal pool - the average number of emigrant propagules per patch in the metapopulation, where $t$ represents the succession of years in the metapopulation (distinct from the age of local populations $\tau$ ). $(1-c) D(t)$ is thus the number of immigrant propagules per patch, under global and uniform dispersal. The dispersal pool depends on the age distribution of local populations and is calculated as

$$
D(t+1)=d \sum_{\tau=0}^{\infty} p_{\tau} \lambda\left(s, N_{\tau}\right) N_{\tau}
$$


Due to competition, survival of these propagules to adulthood in the following year is density dependent. Per-capita survival is thus $C(S)=1 /(1+b S)$, with $1 / b$ being the carrying capacity of each local population (Cheptou 2004). The density of adults $N$ in a population is thus obtained deterministically as

$$
N_{\tau+1}=C\left(S_{\tau}\right) S_{\tau}
$$

with $N_{\tau=0}=0$. Selection depends on the product $a b$ which can therefore be examined as if it is a single parameter for density dependence (shown graphically in Fig. S2b; see also Cheptou 2004, Dornier et al. 2008, and Appendix B). As density dependence $a b$ increases, competition increases because carrying capacity decreases, and cross-fertilisation decreases, especially in local populations with low local density. Metapopulation viability is assessed from the dispersal pool $D(t)$, which provides an overall measure of the metapopulation size (Parvinen 2006, Dornier et al. 2008). If the dispersal pool falls to zero, the metapopulation becomes extinct.

\subsection{Evaluation of mutant fitness}

Given that a single mutant individual with dispersal rate $d^{\prime}$ and selfing rate $s^{\prime}$ establishes in a single local population while the metapopulation is settled at equilibrium, mutant fitness $R\left(d, s, d^{\prime}, s^{\prime}\right)$ is the expected number of surviving mutant offspring equivalents produced during the lifetime of a local population, where two gene copies equals one offspring equivalent (Fisher 1941, Lloyd, 1992 ). While under clonal reproduction, a mutant can invade the resident at equilibrium if the mutant leaves more than one surviving offspring $(R>1)$, for diploid organisms with sexual reproduction, the mutant will invade if it leaves more than two copies of its genes. This is the equivalent to more than one offspring from selfing or more than two offspring from outcrossing (Cheptou 2004). 
If we consider such a newly established mutant in a patch of age $\tau \geq 1$ before reproduction (a mutant individual cannot establish in a patch of age $\tau=0$ since the local population is extinct due to catastrophe), assuming that no new mutant immigrants arrive in the patch (as mutants are extremely rare) and that the mutant population size and the number of mutant propagules produced are always negligible when compared to those of residents, the mutant population dynamics can therefore be described by

$$
\begin{aligned}
& S^{\prime}{ }_{\tau+t}=\left(1-d^{\prime}\right) \lambda^{\prime}\left(s^{\prime}, \bar{N}_{\tau+t}\right) N^{\prime}{ }_{\tau+t} \\
& N^{\prime}{ }_{\tau+t+1}=C\left(\bar{S}_{\tau+t}\right) S^{\prime}{ }_{\tau+t}
\end{aligned}
$$

with $\tau \geq 1, t=0,1,2, \ldots$, and ${N^{\prime}}_{\tau}=1$, as long as local extinction does not occur. Following Lloyd (1992) and Cheptou (2004), the fertility function for calculation of mutant fitness is:

$$
\lambda^{\prime}\left(s^{\prime}, N\right)=f\left(s^{\prime}(1-\delta)+1 / 2\left(1-s^{\prime}\right) A(N)+1 / 2(1-s) A(N)\right) .
$$

This takes into account the effect of the automatic transmission advantage of selfing on fitness (Fisher, 1941): the second term describes the maternal outcrossing contribution where mutant ovules are fertilised by resident sperm/pollen while the third term describes the paternal outcrossing contribution from mutants fertilising resident ovules. In the case that $s^{\prime}=s$, then $\lambda^{\prime}=\lambda$.

The number of emigrants is given by

$$
E_{\tau}=\sum_{t=0}^{\infty} d^{\prime} \lambda^{\prime}\left(s^{\prime}, \bar{N}_{\tau+t}\right) N^{\prime}{ }_{\tau+t}(1-e)^{t}
$$

with $(1-e)^{t}$ the probability that local extinction has not happened in $t$ time steps.

To obtain mutant fitness, we must account for the age distribution $p_{\tau}$ of patches where the mutant can appear, as well as survival of competition and dispersal. Thus, mutant invasion fitness is given by 


$$
R\left(d, s, d^{\prime}, s^{\prime}\right)=(1-c) \sum_{\tau=1}^{\infty} p_{\tau} C\left(\bar{S}_{\tau-1}\right) E(\tau)
$$

As mutant fitness $R\left(d, s, d^{\prime}, s^{\prime}\right)$ includes emigrant mutants produced across multiple years, it is calculated using recursive equations (Appendix A).

Using this formulation of mutant reproduction nevertheless results in an approximation in calculating the invasion fitness, which overestimates the contribution of selfing to fitness. In fact, an accurate diploid model in a metapopulation (Parvinen and Metz 2008) would account for the dominance relationships between mutant and resident alleles, and the frequency of matings between mutant homozygotes, resident homozygotes, and resident-mutant heterozygotes. However, such a model has previously been found not to affect the ESS (Parvinen and Metz 2008), so we do not expect our results to be affected by our approximation. Also, our deterministic model ignores that related individuals may compete (kin competition) and mate with each other (biparental inbreeding) at non-negligible frequencies when local population size is small (Hamilton and May 1977, Perrin and Mazalov 1999, Ravigné et al. 2006). However, we do not expect kin interactions to qualitatively affect findings regarding evolutionary stability of the Good Coloniser Syndrome and the positive versus negative association between selfing and dispersal (see Discussion).

\subsection{Trait evolutionary dynamics}

Evolutionary dynamics of selfing and dispersal rates are described and studied following standard adaptive dynamics procedure (Dieckmann and Law 1996, Metz et al. 1996, Dercole and Rinaldi 2008). Rare mutant individuals characterised by traits $\left(d^{\prime}, s^{\prime}\right)$ are introduced into the metapopulation with resident traits $(d, s)$ at its demographic equilibrium. If the mutants have higher fitness than the residents, the mutant traits become fixed, and thus become the resident traits for the next round of selection. We approximate this stochastic invasion process with the deterministic Ordinary Differential Equations describing the selection This article is protected by copyright. All rights reserved. 
gradients, i.e., the derivative of the invasion fitness with respect to the mutant trait evaluated at the current resident traits,

$$
\begin{aligned}
& \dot{d}=\left.\frac{\partial}{\partial d^{\prime}} R\left(d, s, d^{\prime}, s^{\prime}\right)\right|_{\left(d^{\prime}, s^{\prime}\right)=(d, s)}, \\
& \dot{s}=\left.\frac{\partial}{\partial s^{\prime}} R\left(d, s, d^{\prime}, s^{\prime}\right)\right|_{\left(d^{\prime}, s^{\prime}\right)=(d, s)} .
\end{aligned}
$$

Notice that this assumes independence of mutations and the same mutation rates in the two traits. Correlated mutations and different mutation rates affect the speed of evolution in the two traits. This can affect convergence and evolutionarily stability (Leimar 2001, 2009). However, since we only find evolutionarily and convergence stable node-type equilibria, these assumptions do not affect the results.

\subsection{Numerical analyses}

We study the eco-evolutionary model to assess the effects of environmental and demographic parameters on evolution of dispersal rate $d$ and selfing rate $s$, and on metapopulation extinction or viability. We investigate local extinction rate $e$, density dependence $a b$, cost of dispersal $c$, inbreeding depression $\delta$ and fertility $f$. Simulation outcomes are fully determined by parameter values and starting conditions (i.e. they are deterministic).

Each simulation begins with setting (i) initial values for dispersal rate and selfing rate $(d, s)$, (ii) fixed values for other model parameters; and (iii) an initial size of the dispersal pool $D$. In the initial part of the simulation, the metapopulation is allowed to reach demographic equilibrium, i.e., when $D$ remains constant between generations, $D(t+1)=D(t)=\bar{D}$. This allows us to also obtain the equilibrium of local population size distribution $\bar{N}_{\tau}$ and the equilibrium of propagule distribution $\bar{S}_{\tau}$, i.e., the resident environment to be invaded by mutants. The equilibrium size of the dispersal pool is then evaluated to check whether the metapopulation remains viable or has gone extinct. As in the Dornier et al. (2008), our model 
reveals three ecological scenarios, depending on the set of parameter values and initial selfing and dispersal rates: (1) the metapopulation becomes extinct, regardless of starting density (Unconditional Extinction); (2) the metapopulation is viable, regardless of starting density (Unconditional Viability); and (3) the outcome depends on the starting density, with extinction occurring if the total number of individuals in the metapopulation at the starting point is below a threshold and viable otherwise (Conditional Viability). The Conditional Viability Scenario indicates an emergent Allee effect at the level of the metapopulation (Dornier et al. 2008). In our simulations, we distinguish between these three scenarios for each combination of parameter values and trait values $(d, s)$ by starting simulations from very low $\left(10^{-6}\right)$ and very high $(f / b$, which is maximal dispersal pool size) initial values of $D$ (Dornier et al. 2008). If both cases lead to extinction, this indicates Unconditional Extinction; if both lead to viability, this indicates Unconditional Viability; and if starting at low $D$ leads to extinction but starting at high $D$ leads to viability, this indicates Conditional Viability.

In the second part of the simulation, we compute the selection gradients determining selection on both traits. We numerically integrate such evolutionary trait dynamics with embedded metapopulation dynamics. Such simulations converge to evolutionary singularity where the selection gradient is neutral (slope of the fitness landscape $=0$ ). Evolutionary singularities occur either at fitness maxima, where evolution stops, indicating an Evolutionarily Stable Strategy (ESS) or at fitness minima, when selection is disruptive, leading to evolutionary branching (Geritz et al. 1997, 1998, Della Rossa et al. 2015, Dercole et al. 2016). In our model, evolutionary singularities are always fitness maxima. Thus, the system does not display polymorphism through evolutionary branching. This is because with monotonic density-dependent functions, we can expect monotonic relationships between age and density of local populations, whereas as a non-monotonic relationship is needed for evolutionary branching (Parvinen 1999, 2006). Evolutionary suicide also does not occur: in fact, our model This article is protected by copyright. All rights reserved. 
satisfies the first necessary condition (see Gyllenberg and Parvinen 2001) that the metapopulation can go extinct via a subcritical bifurcation (see grey and dark grey regions in Figures 2 and S1 where the metapopulation transitions from Conditional Viability to Unconditional Extinction). However, as for dispersal, our model violates the second necessary condition for evolutionary suicide. That is, the mutant fitness must depend on the resident strategy explicitly and directly, rather than only depending on the resident strategy through its effect on the metapopulation demographic equilibrium (see section 2.2). Thus, evolutionary suicide by dispersal cannot occur in our model. For selfing, this second necessary condition is also satisfied, so the possibility of evolutionary suicide by selfing cannot be entirely ruled out. Nevertheless, we have not observed the sufficient condition for evolutionary suicide by selfing, i.e. the selection gradient on selfing must point towards the subcritical extinction boundary (see e.g., Figures 2 and S1), nor evolutionary trapping (sensu Ferrière and Legendre 2013) during the sensitivity analyses. However, a different type of extinction (ecological and stochastic) could happen in the Conditional Viability region under stochastic fluctuation in metapopulations size, whenever the metapopulation size would stochastically be disturbed to below the viability threshold. Evolution can lead to this stochastic extinction, when driving traits from the Unconditional Viability to the Conditional Viability region (see this kind of suicide in Rousset and Ronce 2004).

As we did not find alternative evolutionary singularities but always a single global ESS, we therefore discuss how the ESS changes in different environmental and demographic conditions. A detailed explanation of the computation of the relative reproductive fitness (invasion fitness) of the mutant is provided in Appendix A.

As $s$ and $d$ both range from 0 to 1 , they can be visualised on a plane divided into four quadrants separated by the lines for $d=0.5$ and $s=0.5$. We treat these quadrants as 
different evolutionary syndromes: Low Dispersal-Low Selfing (Ld-Ls), low dispersal high selfing (Ld-Hs), High Dispersal-Low Selfing (Hd-Ls) and High Dispersal-High Selfing, which we interpret as the Good Coloniser Syndrome (GCS). We choose 0.5 as a cut off because it is the midpoint of the potential range of both traits in our model. This is reasonable for selfing, which almost always evolves to the boundaries $(0,1)$ in our model and has a similar but continuous bimodal distribution in nature (Jarne and Auld 2006, Moeller et al. 2017). In the context of our model, this is also reasonable for dispersal rate, but in nature, where this trait may be constrained in a smaller but unknown range (for instance through constraints on evolution of dispersal structures), dispersing-selfing syndromes are probably better evaluated in relative terms (see Darling et al. 2008, De Waal et al. 2014).

To explore the likelihood of the various ecological scenarios and evolutionary syndromes with respect to demographic and environmental parameters, we performed four sets of simulations with randomly sampled parameter values and initial trait values of $d=0.5$ and $s=0.5$. To explore a broader range of parameter space, the four sets were obtained combining two different lognormal distributions $\operatorname{LN}($ mean, $\mathrm{SD})$ : $\operatorname{LN}(3,1)$ or $\operatorname{LN}(5,1))$ for $f$ with $(\mathrm{LN}(0,1)$ or $\mathrm{LN}(-3,1)$ for $a b$ (Table $\mathrm{S} 1)$, while always using a uniform distribution between one and zero for $e, c, d$ and $\delta$. As there are potentially many ways to sample random parameter values (Calcagno et al. 2006), this is a somewhat coarse approach, but at least gives qualitatively meaningful results. For each set, we first recorded the ecological scenario at demographic equilibrium without evolution of dispersal and selfing for at least 100 simulations. For 100 simulations that did not start from the Unconditional Extinction scenario, we recorded in which evolutionary syndrome the ESS occurred.

To investigate the effects of variation in parameters on the joint evolution of selfing and dispersal rates and hence the location of the ESS, we performed a sensitivity analysis with 
respect to all possible pairs of parameters, systematically changing them on a discrete grid and recording the position of the ESS. While our model does not allow analytical solutions relating parameters to selection gradients and ESS, our definition of fitness (Section 2.1) nonetheless allows an intuitive interpretation. We also assess how changing parameter values affects selection on selfing and dispersal in a simplified model that omits the distribution of local population density. By assuming that all the non-extinct patches have reached the stationary maximum local population $1 / b$, we are able to obtain insightful analytical solutions (Appendix B).

\section{Results}

\subsection{Ecological and Evolutionary Scenarios}

Simulations with randomly chosen parameter values gave evolutionary endpoints in all four evolutionary syndromes, including the Good Coloniser Syndrome (High Dispersal-High Selfing). These were always ESS. Across the four sets of simulations, 4-20\% of simulations produced the Unconditional Viability scenario, 52-88\% the Conditional Viability scenario, and $5-41 \%$ the Unconditional Extinction scenario. Of simulations not in the Unconditional Extinction scenario, 30-58\% converged to the Good Coloniser Syndrome, 4-31\% to Low Dispersal-High Selfing, 26-54\% to High Dispersal-Low Selfing, and 4-18\% to Low Dispersal-Low Selfing. In these random simulations the ESS for selfing rate was always one or zero (complete selfing or complete outcrossing) but the ESS for dispersal was always intermediate.

We show selected simulations to illustrate how transitory dynamics lead to ESS in all four evolutionary syndromes, including the Good Coloniser Syndrome, depending on parameter values. In these examples the Good Coloniser Syndrome emerges under high extinction rate $e$ with high density dependence $a b$ (Fig. 1) or low inbreeding depression $\delta$ and low cost of This article is protected by copyright. All rights reserved. 
dispersal $c$ (Fig. S1). We also illustrate simulations with different parameter values that all lead to the Good Coloniser Syndrome (Fig. 2). These simulations (Figs 1, 2, S1) also show that extinction is more likely when parameter values reduce local population density across the metapopulation: with increasing values of extinction rate $e$, density dependence $a b$, cost of dispersal $c$, inbreeding depression $\delta$, and decreasing values of fertility $f$ (i.e. the Conditional Viability and Unconditional Extinction scenarios occupy a bigger area of dispersal-selfing space).

\subsection{Selection on selfing and dispersal rates}

When single parameters are changed in sensitivity analyses, the ESS of dispersal rate generally changes gradually, while the ESS of selfing rate remains constant at one and zero for large parameter ranges but switches rapidly between the two extremes when it does change (Figs 3, S2). Thus, although intermediate selfing rates are possible, they only exist for narrow parameter ranges (see Supplementary discussion). The ESS for selfing changes from complete outcrossing (selfing rate of zero) to complete selfing (selfing rate of one) with increasing density dependence $a b$, extinction rate $e$ and cost of selfing $c$ and with decreasing fertility $f$ and inbreeding depression $\delta$ (Figs 3, S2). In the simplified model, the direction of selection on selfing depends only on $\delta, a$, and $b$ (Appendix B), which confirms the effects of these parameters in the full model. The ESS for dispersal increases as fertility $f$ and inbreeding depression $\delta$ increase and as density dependence $a b$ and cost of dispersal $c$

decrease. Trivially, inbreeding depression $\delta$ has no effect on dispersal under complete outcrossing. It is also trivial that $a$ has no effect on selection under complete selfing. Given that selection depends on the product $a b$, this must also be true for $b$ (Fig. 3). A biological reason for $b$ having no effect on the ESS under complete selfing is, however, less obvious. 
Analysis of the simplified model (Appendix B) confirms the patterns observed for $f, \delta, a b$ and $c$ in the sensitivity analysis (Tables S2-S4).

Sensitivity analyses (Fig. 3, S2) show the effects of changing single parameters or pairs of parameters on the ESS for selfing and dispersal. Changing single parameters (moving parallel to the axes) mostly results in opposite changes to the ESS for the two traits, producing a negative relationship between them. The exception is local extinction rate $e$, where both traits increase with increasing values of $e$, creating a positive relationship (Fig. 3a, $e>0.4$, f $>$ 50). Changing two parameters at a time can lead to either positive or negative relationships for most pairs of traits (Figs 3, S2). For instance, increasing extinction rate $e$ and density dependence $a b$ (moving from bottom left to top right in Fig. $3 \mathrm{~b}$ ) creates a positive relationship while increasing one of these parameters and decreasing the other creates a negative relationship. The same holds for inbreeding depression $\delta$ and cost of dispersal $c$ (Fig. 3c).

The effect of the two traits on each other can also be seen in sensitivity analyses. The negative effect of selfing on dispersal can usually be observed as a small decrease in the ESS for dispersal rate when the ESS for selfing rate changes from zero to one (Figs. 3, S2), and analytically in the simplified model (Appendix B). It is harder to discern the impact of dispersal on selfing than vice versa in our sensitivity analyses because the ESS changes much less rapidly for dispersal than selfing (Fig. 3). However, increasing dispersal should be responsible for the decrease in selfing rate from one to zero when extinction rate $e$ increases from low values (Fig. 3a: $e<0.4 f>50$ ), as the effect of $e$ itself on selfing is always positive. 


\section{Discussion}

\subsection{Selection on selfing and dispersal rates}

Selfing has been shown to reduce selection on dispersal for escape from competition because it reduces the influence of the environment on individual reproduction, making propagule production, and therefore competition, more homogeneous (Cheptou and Massol 2009). The negative effect of selfing on dispersal is relatively small in our model (Fig. 3) because heterogeneity in local density ensures heterogeneity in competition, even under complete selfing (i.e. $s=1$ ). Under these conditions, the joint evolution of selfing and dispersal occurs mainly through their independent responses to common selection pressures and we find that the effects of parameters on the ESS (Fig. 3, S2) are generally in line with expectations from the literature (Lloyd 1992, Clobert et al. 2004, Goodwillie et al. 2005, Ronce 2007, Karron et al. 2012, Matthysen 2012). This allows a positive relationship between selfing and dispersal, and the Good Coloniser Syndrome can be an ESS when high values of both traits are selected for simultaneously, for instance under high local extinction (Fig. 3a).

In contrast to our model, in Cheptou and Massol's (2009) model, the benefit of dispersal for escape from competition is strongly reduced under high selfing because all local populations have the same density of adults. By reducing the effect of the environment on per capita propagule production, higher selfing causes greater homogeneity in propagule production, and hence competition, when local density is the same for all patches. Their model therefore only predicts syndromes of no dispersal and high-selfing or dispersal and no selfing, resulting in a negative relationship between the two traits (Cheptou and Massol 2009, Massol and Cheptou 2011, Sun and Cheptou 2012, although see extension by Iritani and Cheptou 2017). 
Incorporating spatiotemporal heterogeneity in density in our model leads to a wider range of predictions and thus broadens our understanding of the joint evolution of selfing and dispersal. However, we did not consider mating between relatives (e.g. siblings), which has significant consequences for evolution of both dispersal and selfing through inbreeding depression and competition (Hamilton and May 1977, Perrin and Mazalov 1999).

Nevertheless, we expect our results to remain accurate where low-density populations still contain relatively large numbers of individuals, or where small local populations are infrequent and thus not important to overall fitness. In other circumstances, relaxing this simplifying assumption would change the parameter ranges leading to the different ecological and evolutionary outcomes. However, we don't foresee that it would alter our main findings that all four syndromes of selfing and dispersal rates, including the Good Coloniser Syndrome, are possible, and that these two traits can be positively or negatively related (see also section 4.3 Model limitations and future directions).

Selection on selfing rate in our model reflects the balance between the negative effect of inbreeding depression $\delta$ and the benefits of the transmission advantage and reproductive assurance (although the transmission advantage benefit does not change with trait and parameter values here, so we do not discuss it further, see, however, Appendix B). The reproductive assurance benefit depends on local density across the metapopulation, which is affected by all parameters. Reducing fertility $f$ and increasing cost of dispersal $c$ and density dependence $a b$ favour selfing by reducing local density, especially in recently recolonised local populations. Increasing local extinction rate $e$ favours selfing by increasing the frequency of recently recolonised local populations with low density. Although inbreeding depression $\delta$ has previously been found to sometimes favour selfing through reproductive assurance by reducing survival and hence local density (Cheptou 2004, Dornier et al., 2008), we only observed the direct negative effect of $\delta$ on the ESS for selfing (Figs 3, S2). The This article is protected by copyright. All rights reserved. 
finding that the ESS for selfing is usually one or zero in our model is broadly consistent with the empirical distribution of selfing rates, which is bimodal with peaks close to zero and one (Jarne and Auld 2006, Moeller et al. 2017). Our results are also similar to those from other models with similar selection pressures on this trait, which only find ESS of one or zero (Cheptou 2004; Dornier et al. 2008, although see Cheptou and Massol 2009).

Selection on dispersal rate in our model reflects the balance between a direct and negative effect of the cost of dispersal $c$ and the positive effect of escape from density-dependent resource competition, consistent with previous models that investigated spatiotemporal heterogeneity in resource competition (Levin 1984, Olivieri et al. 1995, Parvinen 2006). The benefit of escape from competition depends on the frequency distribution of local density, which is affected by all parameters. Changing parameters in ways that increase local density and competition, i.e. increasing fertility $f$ and decreasing inbreeding depression $\delta$ and density dependence $a b$, increases the ESS for dispersal (Figs 3, S2). Similarly, increasing cost of dispersal $c$ reduces local competition by reducing the number propagules entering patches, which presumably reinforces the direct negative effect of $c$ on dispersal. Dispersal rate usually increases with extinction rate $e$ at first but can decrease at high values of $e$ (Figs 3a, S2a) because the benefit of escape from competition declines as $e$ approaches one and zero due to declining heterogeneity in density and hence competition (Ronce et al. 2000, Parvinen 2006).

According to our model, positive relationships, negative relationships, or even no relationship between dispersal and selfing rates are possible, depending on how environmental conditions vary among species or separate metapopulations within species. Sensitivity analyses show that changing a single parameter frequently causes ESS for selfing and dispersal to change in opposite directions, causing a negative relationship. This is mainly because fertility $f$, density 
dependence $a b$, inbreeding depression $\delta$, and cost of dispersal $c$ have opposite effects on selection of these two traits, although the negative effects of the two traits on each other also contribute (Fig. 3, S2). Changing extinction rate $e$ can create a positive relationship between the ESS for selfing and dispersal rates because it has a positive effect on both (Figs 3a, b). Positive relationships between the ESS for selfing and dispersal may also arise on gradients where parameters change simultaneously, for instance, where extinction rate $e$ and density dependence $a b$ both increase (moving bottom left to top right in Fig. 3b) or inbreeding depression $\delta$ and cost of dispersal $c$ both increase (moving bottom left top right in Fig. 3f).

While theory now shows both negative and positive relationships between selfing and dispersal rates are possible (Cheptou and Massol 2009, this study Figs 3, S2) sparse empirical evidence points more to positive relationships. Two studies directly comparing dispersal and selfing rates show positive relationships (De Waal et al. 2014; Darling et al. 2008). In addition, although positive relationships between selfing ability and colonisation success of native and invasive species are generally attributed to the reproductive assurance benefit of selfing following Baker's Law (Grossenbacher et al. 2017, Grossenbacher et al. 2015, Hao et al. 2011; Pyšek et al. 2011, van Kleunen et al. 2008, van Kleunen and Johnson 2007, Stebbins 1957, Baker 1955, although see Price and Jain 1981, Sutherland 2004), a positive relationship between selfing and dispersal could also contribute these patterns. These empirical studies nevertheless provide an imperfect evaluation of theory as they focus on dispersal and selfing ability, whereas theoretical studies evaluate dispersal and selfing rates (Duputié and Massol 2013).

While it has been argued that positive associations between dioecy and fleshy fruit (Renner and Ricklefs 1995, Vamosi et al. 2003) and between dioecy and occurrence on oceanic islands constitute evidence for a negative relationship between self-fertilisation and dispersal 
(Carlquist 1966, Cheptou and Massol 2009, Massol and Cheptou 2011a) these examples are unconvincing. Even though dioecy largely enforces outcrossing, dioecious species may have colonised islands thanks to self-fertilisation, as they are frequently "leaky" (male and female individuals can bear small numbers of flowers of the opposite sex, leading to selfing, Baker, 1967). In addition, it is generally unclear to what extent the higher frequency of dioecy on islands than mainlands is due colonisation by dioecious species rather than faster evolution and diversification of dioecious species on islands (Baker 1967, Baker and Cox 1984, Grossenbacher et al. 2017 although see Schlessman et al. 2014). As for the association between dioecy and fleshy fruit (which disperse further than dry fruit), this could be due to a confounding factor, rather than selection for a high-dispersal low-selfing syndrome. Woody species are both more likely to be dioecious and more likely to have fleshy fruit (Renner and Ricklefs 1995, Vamosi et al. 2003, Moeller et al. 2017). These examples thus highlight the shortage of relevant empirical data to evaluate theoretical predictions on the joint evolution of selfing and dispersal.

\subsection{Spatiotemporal variation in environmental pressures}

Our sensitivity analyses predict how the ESS for selfing and dispersal should vary among species or separate metapopulations within species in response to environmental conditions. In line with Baker's predictions for the characteristics of weeds (Baker, 1965, 1974), our model predicts that species in environments with frequent local extinction are likely to display the Good Coloniser Syndrome, especially if fertility is low (Fig. 3a), or if density dependence is high (Fig. 3b). This also implies that anthropogenic increases in disturbance frequency may cause species to evolve towards the Good Coloniser Syndrome. However, metapopulation extinction is predicted where propagule production and survival are too severely restricted, i.e where local extinction rate, density dependence, inbreeding depression 
and cost of dispersal are high and fertility is low (e.g. Figs 3b, 3c, S2). This could occur through destruction of some patches and degradation, reduction in size and /or more frequent disturbance of remaining ones.

Our model and that of Cheptou and Massol (2009) give insight into how environmental gradients may shape range limits (Sagarin and Gaines 2002, Sun and Cheptou 2012, Hargreaves and Eckert 2014), although this remains tentative as gene flow and spatial sorting, which we did not consider, would also affect the outcome of evolution (Bridle and Vines 2007, Phillips et al. 2008). Our model suggests that a gradient of increasing cost of dispersal towards the range margin would cause a decrease in dispersal (Fig. $3 \mathrm{f}, \mathrm{g}, \mathrm{i}$ ). This could stabilise (pin) range margins, as is predicted with increasing pollinator failure towards the range margin in the Cheptou and Massol model (Sun and Cheptou 2012). In contrast, our model suggests that a gradient of increasing local extinction rate from range centre to range margin could lead to the evolution of the Good Coloniser Syndrome at the range margin (Fig. 3a), favouring range expansion by long distance dispersal, as Baker envisioned for the colonisation of oceanic islands (Baker 1955).

Although increased self-fertilisation ability at the range margin is common for native species (e.g. Moeller 2006, Busch 2005, Barrett et al. 1989), less is known about dispersal ability in relation to range position. Both selfing and dispersal ability increase towards the range margin in Abronia umbellata (Darling et al. 2008) but no pattern in either trait in relation to range position was found among a suite of annual daisy species (de Waal et al 2014). In invasive species, an increase in both dispersal and selfing towards the invasion front could be expected both due to spatial sorting over repeated colonisation events (Burton et al. 2010, Perkins et al. 2013, Williams et al. 2016, Hui and Richardson 2017, Ochocki and Miller 2017) and due to conventional selection, for instance because of decreasing density and 
increasing heterogeneity in density from the core to the invasion front (Simmons and Thomas, 2004). Thus far, empirical studies have shown evolution of increased dispersal (Simmons and Thomas 2004, Phillips et al. 2006, 2008, 2010, Monty and Mahy 2010, Berthouly-Salazar et al. 2012), but not selfing (Colautti et al. 2010) at the invasion front, (although see Petanidou et al. 2012).

\subsection{Model limitations and future directions}

As a simplifying assumption, we did not consider how competition and mating between kin, which is especially prevalent in small local populations (Ravigné et al. 2006), would affect evolution . If this assumption was relaxed, selection on dispersal would likely increase, both to avoid competing with kin (Hamilton and May 1977) and mating with them (biparental inbreeding), assuming there is inbreeding depression (Perrin and Mazalov 1999). However, the overall effect of biparental inbreeding on selfing depends on genetic structure and magnitude of inbreeding depression, as it has a positive effect by reducing the inbreeding depression cost of selfing and a negative effect by reducing the transmission advantage (Solbrig 1976, Lloyd 1979, Uyenoyama 1986, Ronfort and Couvet 2009). We therefore cannot exclude the possibility that under low local population size, biparental inbreeding might counteract the positive effect of reproductive assurance on selection for selfing. Exploring the implications of kin interactions for the joint evolution of dispersal and selfing is thus a potentially rewarding avenue for future research.

More detail on a number of other processes, in addition to kin interactions, would help us to understand when the different syndromes should evolve, and whether evolutionary branching or stable polymorphism are possible. Including extinction due to ecological succession (Comins, 1980, Olivieri et al. 1995, Ronce et al. 2005) and stochasticity in reproduction and survival within local populations (Parvinen et al. 2003, Cadet et al. 2005) can both increase 
selection for dispersal (the latter especially when population size is small). Moreover, inbreeding depression affects selection on dispersal positively, through avoidance of inbreeding, and negatively, by reducing selection for avoidance of kin competition (Roze and Rousset, 2005). Allowing selection to reduce inbreeding depression (by removing deleterious recessive alleles) would make it easier for selfing to evolve (Dornier et al. 2008, Massol and Cheptou 2011a). Our model also ignores that inbreeding depression is not a purely genetic trait but depends on the environment. Both positive and negative density dependence of inbreeding depression are possible, and would alter how parameters affect evolution of selfing, and provide an additional feedback from dispersal to selfing (Cheptou and Donohue 2011). However, allowing perenniality or a propagule bank (dormant propagules) could reduce selection for selfing by increasing local population density across the metapopulation (Pannell and Barret 1998). In models with kin competition, heterogeneity in patch size and carrying capacity has been found to allow dispersal polymorphism, particularly when there are many small and few large populations (Leturque and Rousset 2002, Massol et al. 2011, Kisdi 2016) and this effect could potentially allow the Good Coloniser Syndrome to exist in stable polymorphic (meta-)populations (Massol and Cheptou 2011, Laroche et al. 2016). Our results suggest that a scenario where habitat patches vary in the frequency of disturbance might also produce a stable polymorphism, with the Good Coloniser Syndrome dominating in more frequently disturbed patches.

\subsection{Conclusions}

In our demographically structured metapopulation model, a positive relationship between dispersal and selfing is a likely outcome of evolution due to spatiotemporal heterogeneity in local density, which promotes evolution of dispersal for escape from competition, and evolution of selfing for reproductive assurance against mate limitation. Consequently, the 
Good Coloniser Syndrome can be selected under relatively high local extinction rates, provided that inbreeding depression and the cost of dispersal are not excessive. This contrasts to previous investigations that predict a strictly negative relationship between selfing and dispersal and no evolution of the Good Coloniser Syndrome (Cheptou and Massol 2009, Massol and Cheptou 2011, Sun and Cheptou 2012). Thus, by showing that both positive and negative relationships are possible, depending on context, our model extends our understanding of the joint evolution of these traits and expands theory to accommodate a wider range of empirical patterns. Also, in considering selection on selfing from mate limitation, it provides a theoretical basis for understanding the joint evolution of selfing and dispersal in animals, whereas previous theory (Cheptou and Massol 2009, Massol and Cheptou 2011, Sun and Cheptou 2012) was only applicable to plants. Nevertheless, as very few empirical studies directly investigate joint evolution of selfing and dispersal (Darling et al. 2008, De Waal et al. 2014), much more empirical work will be needed to allow an adequate evaluation of theoretical predictions.

\section{References}

Auld, J. R., and R. R. de Casas. 2013. The Correlated Evolution of Dispersal and MatingSystem Traits. Evolutionary Biology 40:185-193.

Baker, H. G. 1955. Self-compatibility and establishment after "long-distance" dispersal. Evolution 9:347-349.

Baker, H. G. 1965. Characteristics and Modes of Origin of Weeds. Pages 147-172 in H. G. Baker and G. L. Stebbins, editors. Genetics of Colonizing Species. Academic Press, New York. 
Baker, H. G. 1967. Support for Baker's Law - as a rule. Evolution 21:853-856.

Baker, H. G. 1974. The evolution of weeds. Annual Review of Ecology and Systematics 5:124.

Baker, H. G., and P. A. Cox. 1984. Further Thoughts on Dioecism and Islands. Annals of the Missouri Botanical Garden 71:244-253.

Barrett, S. C. H. 2010. Understanding plant reproductive diversity. Philosophical transactions of the Royal Society of London. Series B, Biological sciences 365:99-109.

Barrett, S. C. H., and D. Charlesworth. 1991. Effects of a change in the level of inbreeding on the genetic load. Nature 353: 522-524.

Bawa, K. S. 1980. Evolution of dioecy in flowering plants. Annual Review of Ecology and Systematics 11:15-39.

Barrett, S. C. H., and J. R. Pannell. 1999. Metapopulation dynamics and mating-system evolution in plants. Pages 74-100 in P. Hollingsworth, R. Bateman, and R. Gornall, editors. Molecular Systematics and Plant Evolution. Chapman and Hall, London.

Barrett S. C .H., M. T. Morgan, and B. C. Husband. 1989. The dissolution of a complex polymorphism: the evolutionof self-fertilization in tristylous Eichhornia paniculata (Pontederiaceae). Evolution 43:1398-1416.

Berthouly-Salazar, C., B.J. van Rensburg, J.J. le Roux, B.J. van Vuuren, and C. Hui 2012. Spatial sorting drives morphological variation in the invasive bird, Acridotheres tristis. PLoS ONE, 7:e38145. 
Berthouly-Salazar, C., C. Hui, T.M. Blackburn, C. Gaboriaud, B.J. van Rensburg, B.J. van Vuuren, and J.J. Le Roux, J.J. 2013. Long-distance dispersal maximizes evolutionary potential during rapid geographic range expansion. Molecular Ecology, 22:5793-5804.

Bridle, J. R., and T. H. Vines. 2007. Limits to evolution at range margins: when and why does adaptation fail? Trends in Ecology and Evolution 22:140-147.

Burton, O. J., B. L. Phillips, and J. M. Travis. 2010. Trade-offs and the evolution of lifehistories during range expansion. Ecology Letters 13:1210-1220.

Busch, J. W. 2005. The evolution of self-compatibility in geographically peripheral populations of Leavenworthia alabamica (Brassicaceae). American Journal of Botany 92:1503-1512.

Busch, J. W. 2011. Demography, Pollination and Baker's Law. Evolution 65:1511-1513.

Cadet, C., R. Ferrière, J. A. Metz, and M. van Baalen. 2005. The Evolution of Dispersal under Demographic Stochasticity. The American Naturalist 162.

Calcagno, V., N. Mouquet, P. Jarne, and P. David. 2006. Coexistence in a metacommunity: the competition-colonization trade-off is not dead. Ecology Letters 9:897-907.

Carlquist, S. 1966. The biota of long-distance dispersal. IV. Genetic systems in the floras of oceanic islands. Evolution 4:433-455.

Charlesworth, D., and B. Charlesworth. 1987. Inbreeding depression and its evolutionary consequences. Annual Review of Ecology and Systematics 18: 237-268.

Charlesworth, B., and D. Charlesworth. 1999. The genetic basis of inbreeding depression. Genetical Research 74:329-340. 
Charlesworth, D., and J. H. Willis. 2009. The genetics of inbreeding depression. Nature Reviews Genetics 10:783-796.

Cheptou, P.-O. 2004. Allee Effect and Self-Fertilization in Hermaphrodites: Reproductive Assurance in Demographically Stable Populations. Evolution 58:2613-2621.

Cheptou, P.-O. 2012. Clarifying Baker's Law. Annals of Botany 109:633-641.

Cheptou, P. O., and K. Donohue. 2011. Environment-dependent inbreeding depression: its ecological and evolutionary significance. New Phytologist 189:395-407.

Cheptou, P.-O. and F. Massol. 2009. Pollination Fluctuations Drive Evolutionary Syndromes Linking Dispersal and Mating System. The American Naturalist 174:46-55.

Clobert, J., R. A. Ims, and F. Rousset. 2004. Causes, mechanisms and consequences of dispersal. Pages 307-335 in I. A. Hanski and O. E. Gaggiotti, editors.Ecology, Genetics and Evolution of Metapopulations. Academic Press, Burlington, USA.

Colautti, R. I., N. A. White, and S. C. H. Barrett. 2010. Variation of self-incompatibility within invasive populations of purple loosestrife (Lythrum salicaria L.) from eastern North America. International Journal of Plant Sciences 171:158-166.

Comins, H.N., W. D. Hamilton, and R. M. May. 1980. Evolutionarily stable dispersal strategies. Journal of Theoretical Biology 82:205-230.

Crnokrak, P., and S. C. H. Barrett. 2002. Perspective: Purging the genetic load: A review of the experimental evidence. Evolution 56:2347-2358.

Darling, E., K. E. Samis, and C. G. Eckert. 2008. Increased seed dispersal potential towards geographic range limits in a Pacific coast dune plant. New Phytologist 178:424-435. 
Darwin, R. C. 1876. The effects of cross and self-fertilization in the vegetable kingdom. John Murray, London.

Della Rossa, F., F. Dercole, and P. Landi. 2015. The Branching Bifurcation of Adaptive Dynamics. International Journal of Bifurcation and Chaos 25:1540001.

Duputié, A., and F. Massol. 2013. An empiricist's guide to theoretical predictions on the evolution of dispersal. Interface Focus 3:20130028.

De Waal, C., J. G. Rodger, B. Anderson, and A. G. Ellis. 2014. Selfing ability and dispersal are positively related, but not affected by range position: A multispecies study on southern African Asteraceae. Journal of Evolutionary Biology 27:950-959.

Dercole, F., and S. Rinaldi. 2008. The Adaptive Dynamics approach and its applications. Princeton University Press.

Dercole, F., F. Della Rossa, and P. Landi. 2016. The transition from evolutionary stability to branching: A catastrophic evolutionary shift. Scientific reports 6:26310.

Dieckmann, U., and R. Law. 1996. The Dynamical Theory of Coevolution: a Derivation from Stochastic Ecological Processes. Journal of Mathematical Biology 34:579-612.

Dornier, A., F. Munoz, and P.-O. Cheptou. 2008. Allee effect and self-fertilization in hermaphrodites: Reproductive assurance in a structured metapopulation. Evolution 62:25582569.

Eckert, C. G., K. E. Samis, and S. Dart. 2006. Reproductive assurance and the evolution of uniparental reproduction in flowering plants. Pages 183-203in L. D. Harder and S. C. H. Barrett, editors.Ecology and evolution of flowers. Oxford University Press, New York. 
Ferrière, R. and Legendre, S. 2013. Eco-evolutionary feedbacks, adaptive dynamics and evolutionary rescue theory. Philosophical Transactions of the Royal Society of London B:

Biological Sciences 368:20120081

Fisher, R. A. 1941. Average excess and average effect of a gene substitution. Annals of Eugenics 11:53-63.

Gascoigne, J., L. Berec, S. Gregory, and F. Courchamp. 2009. Dangerously few liaisons: a review of mate-finding Allee effects. Population Ecology 51:355-372.

Geritz, S. A. H., É. Kisdi, G. Meszéna, and J. A. J. Metz. 1998. Evolutionarily Singular Strategies and the Adaptive Growth and Branching of the Evolutionary Tree. WP-96-114. Evolutionary Ecology 12:35-37.

Geritz, S. A. H., J. A. J. Metz, É. Kisdi, and G. Meszena. 1997. Dynamics of adaptation and evolutionary branching. Physical Review Letters 78:2024-2027.

Goodwillie, C., S. Kalisz, and C. G. Eckert. 2005. The evolutionary enigma of mixed mating systems in plants: occurrence, theoretical explanations and empirical evidence. Annual Review of Ecology, Evolution and Systematics 36:47-49.

Grossenbacher, D., R. B. Runquist, E. E. Goldberg, and Y. Brandvain. 2015. Geographic range size is predicted by mating system. Ecology Letters 18:706-713.

Grossenbacher, D. L., Y. Brandvain, J. R. Auld, M. Burd, P. O. Cheptou, J. K. Conner, A. G. Grant, S. M. Hovick, J. R. Pannell, A. Pauw, T. Petanidou, A. M. Randle, R. Rubio de Casas, J. Vamosi, A. Winn, B. Igic, J. W. Busch, S. Kalisz, and E. E. Goldberg. 2017. Selfcompatibility is over-represented on islands. New Phytologist 215:469-478.

Hamilton, W.D. and R. M. May. 1977. Dispersal in stable habitats. Nature 269:578-581. 
Hao, J.H., S. Qiang, T. Chrobock, M. Van Kleunen, and Q.Q. Liu. 2011. A test of Baker's law: breeding systems of invasive species of Asteraceae in China. Biological Invasions, 13, $571-580$.

Hanski, I. 1998. Metapopulation dynamics Nature 396: 41-49.

Hastings, A. 1983. Can spatial variation alone lead to selection for dispersal. Theoretical Population Biology 24:244-251.

Hargreaves, A. L., and C. G. Eckert. 2014. Evolution of dispersal and mating systems along geographic gradients: Implications for shifting ranges. Functional Ecology 28:5-21.

Hui, C. and D. M. Richardson. 2017. Invasion Dynamics. Oxford University Press, Oxford.

Husband, B. C., and D. W. Schemske. 1996. Evolution of the magnitude and timing of inbreeding depression in plants. Evolution 50:54-70.

Iritani, R. and P.-O. Cheptou. 2017. Joint evolution of differential seed dispersal and selffertilization. Journal of Evolutionary Biology 30: 1526-1543.

Jarne, P., and J. R. Auld. 2006. Animals mix it up too: the distribution of self-fertilization among hermaphroditic animals. Evolution 60:1816-1824.

Karron, J. D., C. T. Ivey, R. J. Mitchell, M. R. Whitehead, R. Peakall, and A. L. Case. 2012. New perspectives on the evolution of plant mating systems. Annals of Botany 109:493-503.

Kisdi, E. 2016. Dispersal polymorphism in stable habitats. Journal of Theoretical Biology 392:69-82.

Kubisch, A., R. D. Holt, H. J. Poethke, and E. A. Fronhofer. 2014. Where am I and why? Synthesizing range biology and the eco-evolutionary dynamics of dispersal. Oikos 123:5-22. 
Lande, R., and E. Porcher. 2015. Maintenance of quantitative genetic variance under partial self-fertilization, with implications for evolution of selfing. Genetics 200:891-906.

Lande, R., and D. W. Schemske. 1985. The evolution of self-fertilization and inbreeding depression in plants. I. Genetic models. Evolution 39:24-40.

Laroche, F., P. Jarne, T. Perrot, and F. Massol. 2016. The evolution of the competitiondispersal trade-off affects alpha- and beta-diversity in a heterogeneous metacommunity. Proceedings of the Royal Society B: Biological Sciences 283.

Leimar, O. 2001. Evolutionary change and Darwinian demons. Selection 2:65-72.

Leimar, O. 2009. Multidimensional convergence stability. Evolutionary Ecology Research 11:191-208.

Leimu, R., P. Mutikainen, J. Koricheva and M. Fischer. 2006. How general are positive relationships between plant population size, fitness and genetic variation? Journal of Ecology 94:942-952.

Leturque, H., and F. Rousset. 2002. Dispersal, kin competition, and the ideal free distribution in a spatially heterogeneous population. Theoretical Population Biology 62:169-180.

Levin, S. A., D. Cohen, and A. Hastings. 1984. Dispersal strategies in patch environments. Theoretical Population Biology 26:165-191.

Levins, R. 1969. Some demographic and genetic consequences of environmental heterogeneity for biological control. Bulletin of the Entomological Society of America $15: 237-240$.

Lloyd, D. G. 1979. Some reproductive factors affecting the selection of self-fertilization in plants. The American Naturalist 113:67-79. 
Lloyd, D. G. 1992. Self-fertilization and cross-fertilization in plants 2. The selection of selffertilization. International Journal of Plant Sciences 153:370-380.

Massol, F., and P.-O. Cheptou. 2011a. Evolutionary syndromes linking dispersal and mating system: the effect of autocorrelation in pollination conditions. Evolution 65:591-598.

Massol, F., and P.-O. Cheptou. 2011b. When should we expect the evolutionary association of self-fertilization and dispersal? Evolution 65:1217-1220.

Massol, F., and F. Débarre. 2015. Evolution of dispersal in spatially and temporally variable environments: The importance of life cycles. Evolution 69:1925-1937.

Massol, F., A. Duputié, P. David, and P. Jarne. 2011. Asymmetric patch size distribution leads to disruptive selection on dispersal. Evolution 65:490-500.

Matthysen, E. 2012. Multicausality of dispersal: a review. Pages 3-18 in J. Clobert, M. Baguette, T. G. Benton, J. M. Bullock, and S. Ducatez, editors. Dispersal ecology and evolution. Oxford University Press, Oxford, UK.

McPeek, M. A., and R. D. Holt. 1984. The evolution of dispersal in spatially temporally varying environments. The American Naturalist 140:1010-1027.

Metz, J. A. J., S. A. H. Geritz, G. Meszena, F. J. A. Jacobs, and J. van Heerwaarden. 1996. Adaptive dynamics: a geometrical study of the consequences of nearly faithful reproduction. Pages 183-231 in S. J. van Strien and S. M. Verduyn Lunel, editors. Stochastic and Spatial Structures of Dynamical Systems. Elsevier, Amsterdam.

Moeller, D. A. 2006 Geographic structure of pollinator communities, reproductive assurance, and the evolution of self-pollination. Ecology 87:1510-1522 
Moeller, D. A., R. D. Briscoe Runquist, A. M. Moe, M. A. Geber, C. Goodwillie, P.-O. Cheptou, C. G. Eckert, E. Elle, M. O. Johnston, S. Kalisz, R. H. Ree, R. D. Sargent, M. Vallejo-Marin, and A. A. Winn. 2017. Global biogeography of mating system variation in seed plants. Ecology Letters 20: 375-384.

Monty, A., and G. Mahy. 2010. Evolution of dispersal traits along an invasion route in the wind-dispersed Senecio inaequidens (Asteraceae). Oikos 119:1563-1570.

Morgan, M. T., and W. G. Wilson. 2005. Self-fertilization and the escape from pollen limitation in variable pollination environments. Evolution 59:1143-1148.

Motro, U. 1991. Avoiding inbreeding and sibling competition: the evolution of sexual dimorphism for dispersal. The American Naturalist 137:108-115.

Ochocki, B. M., and T. E. X. Miller. 2017. Rapid evolution of dispersal ability increases the speed and variability of biological invasions. Nature Communications 8:14315.

Olivieri, I., Y. Michalakis, and P. H. Gouyon. 1995. Metapopulation genetics and the evolution of dispersal. The American Naturalist 146:202-228.

Pannell, J. R. 2015. Evolution of the mating system in colonizing plants. Molecular Ecology 24:2018-2037.

Pannell, J. R., J. R. Auld, Y. Brandvain, M. Burd, J. W. Busch, P. Cheptou, J. K. Conner, E. E. Goldberg, A. Grant, D. L. Grossenbacher, S. M. Hovick, B. Igic, S. Kalisz, T. Petanidou, A. M. Randle, R. R. De Casas, A. Pauw, and J. C. Vamosi. 2015. The scope of Baker's law. New Phytologist 208:656-667.

Pannell, J. R., and S. C. H. Barrett. 1998. Baker's law revisited: Reproductive assurance in a metapopulation. Evolution 52:657-668. 
Parvinen, K. 2006. Evolution of dispersal in a structured metapopulation model in discrete time. Bulletin of Mathematical Biology 68:655-678.

Parvinen, K. 1999. Evolution of migration in a metapopulation. Bulletin of Mathematical Biology 61:531-550.

Parvinen, K., U. Dieckmann, M. Gyllenberg, and J. A. Metz. 2003. Evolution of dispersal in metapopulations with local density dependence and demographic stochasticity. Journal of Evolutionary Biology 16:143-153.

Parvinen, K., and J. A. J. Metz. 2008. A novel fitness proxy in structured locally finite metapopulations with diploid genetics, with an application to dispersal evolution. Theoretical Population Biology 73:517-528.

Perkins, A. T., B. L. Phillips, M. L. Baskett, and A. Hastings. 2013. Evolution of dispersal and life history interact to drive accelerating spread of an invasive species. Ecology Letters $16: 1079-1087$.

Perrin, N. and V. Mazalov. 1999. Dispersal and inbreeding avoidance. The American Naturalist 154:282-292.

Petanidou, T., R. C. Godfree, D. S. Song, A. Kantsa, Y. L. Dupont, and N. M. Waser. 2012. Self-compatibility and plant invasiveness: Comparing species in native and invasive ranges. Perspectives in Plant Ecology Evolution and Systematics 14:3-12.

Phillips, B. L., G. P. Brown, and R. Shine. 2010. Evolutionarily accelerated invasions: The rate of dispersal evolves upwards during the range advance of cane toads. Journal of Evolutionary Biology 23:2595-2601. 
Phillips, B. L., G. P. Brown, J. M. J. Travis, and R. Shine. 2008. Reid's paradox revisited: the evolution of dispersal kernels during range expansion. The American naturalist 172:S34-S48.

Phillips, B. L., G. P. Brown, J. K. Webb, and R. Shine. 2006. Invasion and the evolution of speed in toads. Nature 439:803.

Price, S. C., and S. K. Jain. 1981. Are inbreeders better colonizers? Oecologia 49:283-286.

Pyšk, P., V. Jarošík, M.Chytrý, J.Danihelka, I. Kühn, J. Pergl, L.Tichý, J.C. Biesmeijer, W.N. Ellis, W.E. Kunin, and J. Settele, 2011. Successful invaders co-opt pollinators of native flora and accumulate insect pollinators with increasing residence time. Ecological Monographs, 81: 277-293.

Ravigné, V., Olivieri, I., González-Martínez, S.C. and Rousset, F. 2006. Selective interactions between short-distance pollen and seed dispersal in self-compatible species. Evolution 60:2257-2271.

Renner, S. S., and R. E. Ricklefs. 1995. Dioecy and its correlates in the flowering plants. American Journal of Botany 82:596-606.

Robinet, C. and Liebhold, A. M. 2009. Dispersal polymorphism in an invasive forest pest affects its ability to establish. Ecological Applications 19:1935-1943.

Ronce, O. 2007. How does it feel to be like a rolling stone? Ten questions about dispersal evolution. Annual Review of Ecology, Evolution, and Systematics 38:231-253.

Ronce, O., F. Perret and I. Olivieri 2000. Evolutionarily Stable Dispersal Rates Do Not Always Increase with Local Extinction Rates. The American Naturalist 155:485-496.

Ronfort, J., and D. Couvet. 2009. A stochastic model of selection on selfing rates in structured populations. Genetical Research 65 . 
Roques, L., J. Garnier, F. Hamel, and E. K. Klein. 2012. Allee effect promotes diversity in traveling waves of colonization. Proc Natl Acad Sci U S A 109:8828-8833.

Roze, D., and F. Rousset. 2005. Inbreeding Depression and the Evolution of Dispersal Rates: A Multilocus Model. The American Naturalist 166:708-721.

Sagarin, R. D., and S. D. Gaines. 2002. The "abundant centre" distribution: to what extent is it a biogeographical rule? Ecology Letters 5:137-147.

Schlessman, M. A., L. B. Vary, J. Munzinger, and P. P. Lowry. 2014. Incidence, correlates, and origins of dioecy in the island flora of New Caledonia. International Journal of Plant Sciences 175:271-286.

Simmons, A. D., and C. D. Thomas. 2004. Changes in dispersal during species' range expansions. The American Naturalist 164:378-395.

Solbrig, O. T. 1976. On the Relative Advantages of Cross-and Self-Fertilization. Annals of the Missouri Botanical Garden 63:262-276.

Stebbins, G. L. 1957. Self fertilization and population variability in higher plants. The American Naturalist 41:337-354.

Stephens, P. A., W. J. Sutherland, and R. P. Freckleton. 1999. What is an Allee effect? Oikos 87:185-190.

Sun, S., and P.-O. Cheptou. 2012. Life-history traits evolution across distribution ranges: how the joint evolution of dispersal and mating system favor the evolutionary stability of range limits? Evolutionary Ecology 26:771-778.

Sutherland, S. 2004. What makes a weed a weed: life history traits of native and exotic plants in the USA. Oecologia 141:24-39. 
Uyenoyama, M. K. 1986. Inbreeding and the cost of meiosis: the evolution of selfing in populations practicing biparental inbreeding. Evolution 40:388-404.

Vamosi, J. C., S. P. Otto, and S. C. H. Barrett. 2003. Phylogenetic analysis of the ecological correlates of dioecy in angiosperms. Journal of Evolutionary Biology 16:1006-1018.

van Kleunen, M. and S. D, Johnson. 2007. Effects of self-compatibility on the distribution range of invasive European plants in North America. Conservation Biology 21:1537-1544.

van Kleunen, M., J. C. Manning, V. Pasqualetto, and S. D. Johnson. 2008. Phylogenetically independent associations between autonomous self-fertilization and plant invasiveness.

American Naturalist 171:195-201.

van Valen, L. 1971. Group Selection and the Evolution of Dispersal. Evolution 25:591-598.

Williams, J. L., B. E. Kendall, and J. M. Levine. 2016. Experimental Landscapes. Science $353: 482-485$.

Wright, S. 1931. Evolution in Mendelian populations. Genetics 16:97-159.

Wright, S. 1977. Evolution and the genetics of populations, vol. 3. Experimental results and evolutionary deductions. University of Chicago Press, Chigago, IL.

Figure Legends: Fig. 1. Consequences of extinction rate $e$ and density dependence $a b$ for evolution of dispersal rate $d$ (horizontal axis) and selfing rate $s$ (vertical axis) and for viability versus extinction of the metapopulation. Lines represent the path of dispersal and selfing rate evolution from different starting values. Filled circles represent (globally stable 
and unique) evolutionarily stable strategies (ESS). Shading denotes ecological scenarios:

white for the Unconditional Viability scenario, dark grey for the Unconditional Extinction scenario, and grey for the Conditional Viability scenario. Moving from left to right, extinction rate $e$ increases (from 0.25 to 0.5 ), causing an increase in dispersal rate. Moving from bottom to top, density dependence increases (from 0.01 to 0.1 ), causing an increase in selfing rate (from 0 to 1 ). Other parameter values are cost of dispersal $c=0.5$, inbreeding depression $\delta=0.55$, fertility $f=20$.

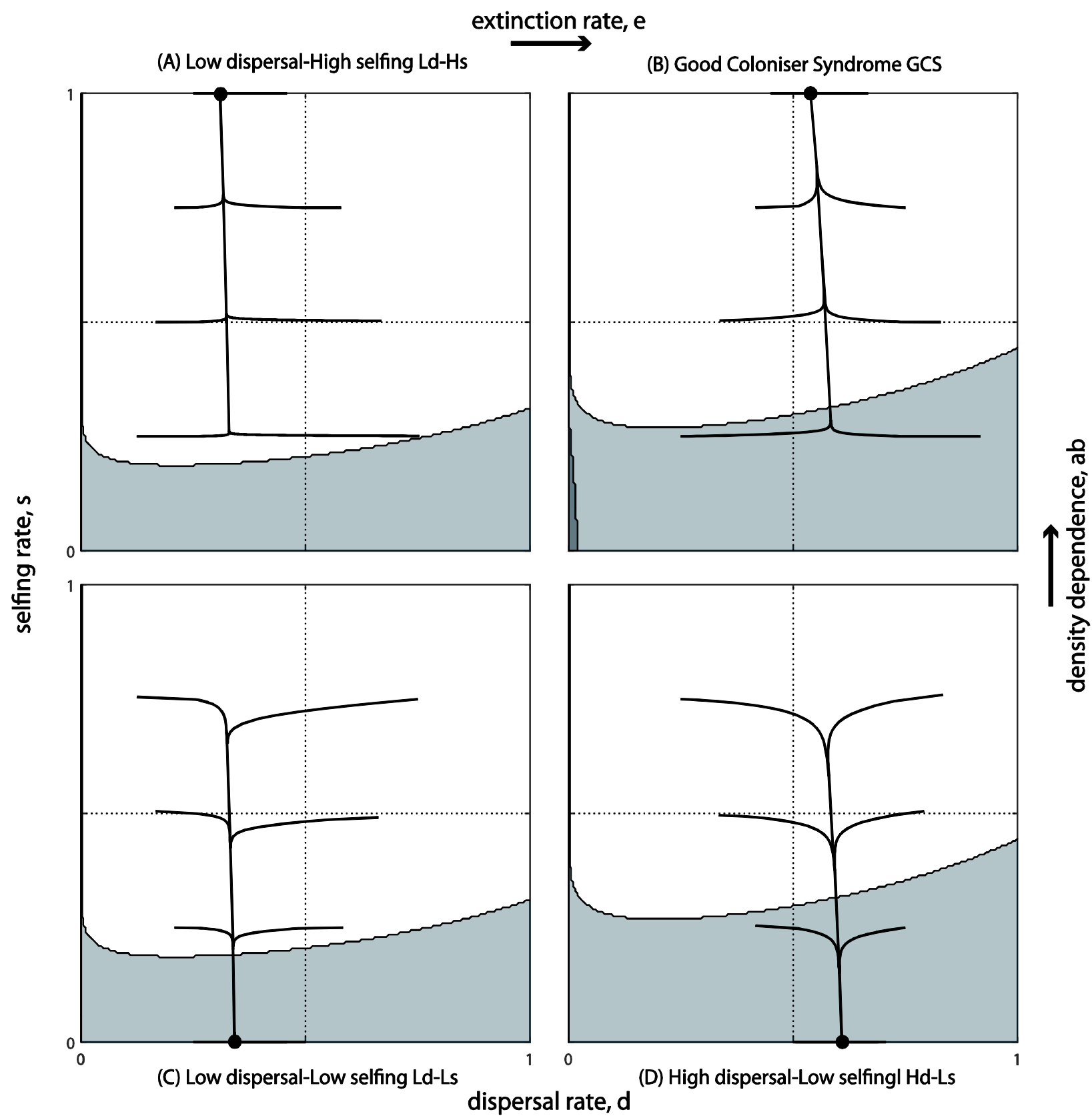

This article is protected by copyright. All rights reserved. 
Fig. 2. Simulations for different sets of parameters that lead to the Good Coloniser Syndrome as an ESS. Dispersal rate $d$ is shown on the horizontal axis and selfing rate $s$ on the vertical axis. Lines represent the path of dispersal and selfing rate evolution from different starting values. Filled circles represent (globally stable and unique) evolutionarily stable strategies (ESS). Shading denotes ecological scenarios: white for the Unconditional Viability scenario, dark grey for the Unconditional Extinction scenario and grey for the Conditional Viability scenario. Density dependence $a b$ increases from 0.1 in (A) to 1 in (B), fertility $f$ increases from 20 in (B) to 100 in (D), and extinction rate $e$ increases from 0.75 in (D) to 0.9 in (C). Other parameter values are cost of dispersal $c=0.5$, inbreeding depression $\delta=0.55$.

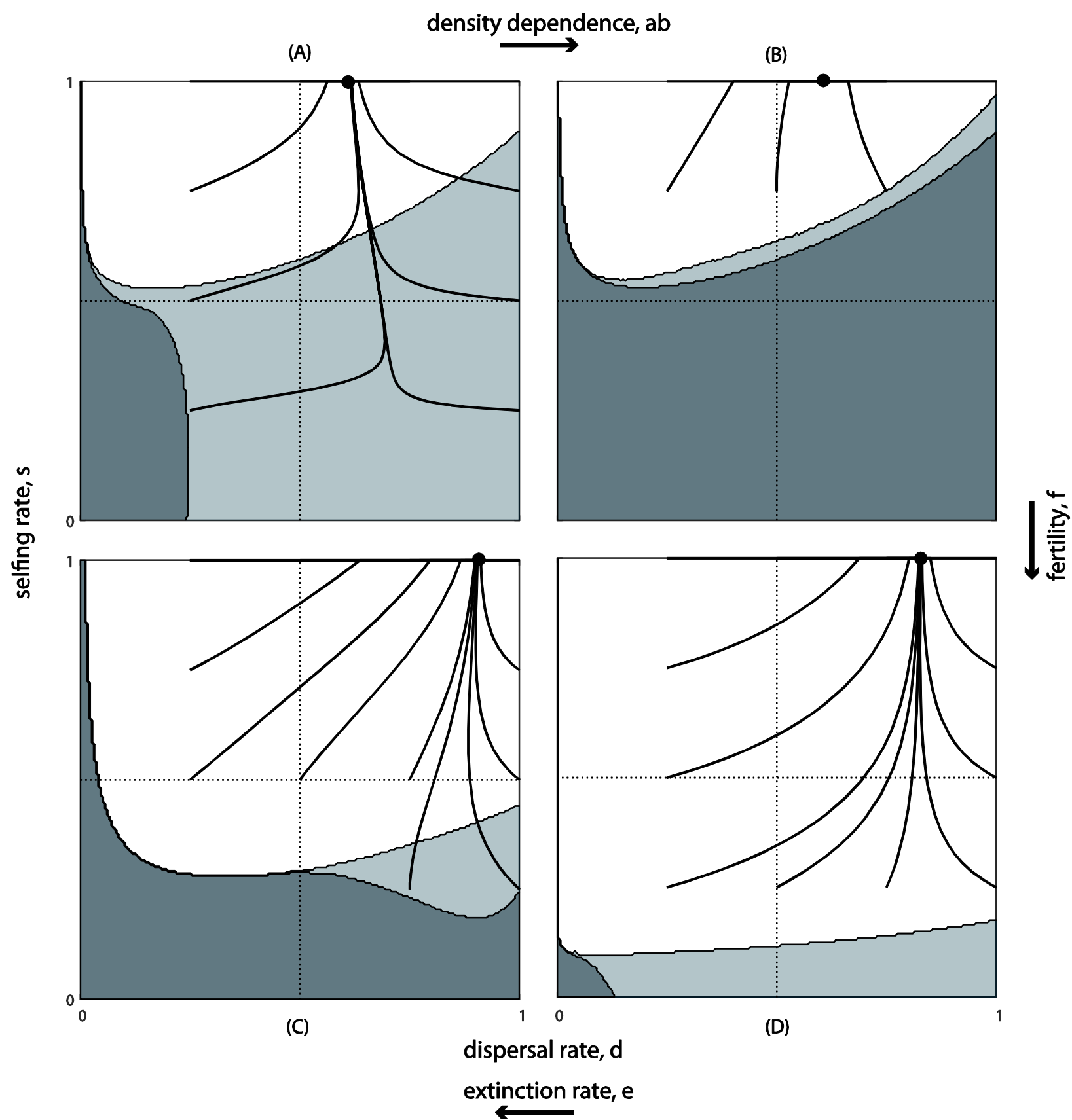

This article is protected by copyright. All rights reserved. 
Fig. 3. Sensitivity analyses showing the effect of varying two parameter values on the ESS for dispersal and selfing rate. Boundaries between areas of different colour shading indicate 0.1 contours of dispersal rate, ascending from cold to hot colours. Coloured lines represent 0.1 contours of selfing rate, ascending from cold to hot colours. Black lines represent the boundaries between the four evolutionary syndromes. Grey areas represent unviable metapopulations. Hd-Ls = High Dispersal-Low Selfing, Ld-Hs = Low Dispersal-High Selfing, Ld-Ls = Low Dispersal-Low Selfing and GCS = Good Coloniser Syndrome (High Dispersal-High Selfing). Other parameter values are density dependence $a b=0.1$, cost of dispersal $c=0.5$, inbreeding depression $\delta=0.55$, extinction rate $e=0.5$, fertility $f=20$.
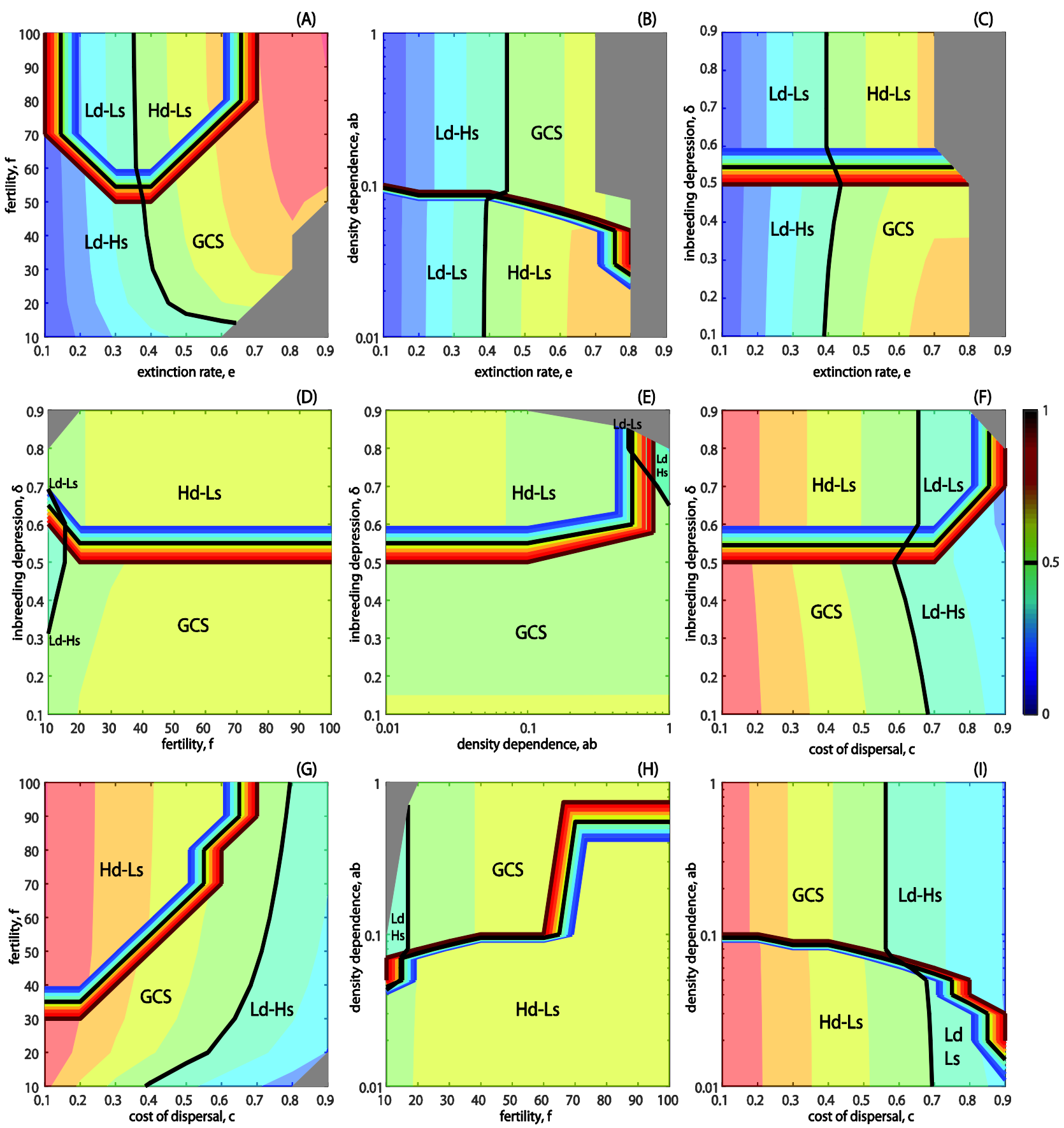

This article is protected by copyright. All rights reserved. 


\section{Appendix}

\section{Appendix A. Evaluation of mutant fitness}

Following the approach of Parvinen (2006), the number of emigrant propagules can be computed recursively, i.e., the per-capita number of emigrants produced by a mutant establishing in a patch of age $\tau$ is given by two contributions: the first is the number of propagules directly dispersed by that mutant, while the second represents the propagules that stay in the patch. If they survive competition and local extinction, they can be considered as mutants appearing in a patch of age $\tau+1$, which in turn will produce emigrant propagules. In formula, we have

$$
E_{\tau}=d^{\prime} \lambda^{\prime}\left(s^{\prime}, \bar{N}_{\tau}\right)+\left(1-d^{\prime}\right) \lambda^{\prime}\left(s^{\prime}, \bar{N}_{\tau}\right) C\left(\bar{S}_{\tau}\right)(1-e) E_{\tau+1}
$$

If we assume that the population dynamics has converged to its attractor, we can practically set an upper limit $L$ (10 in the present paper) for the age $\tau$ of the patches so that

$$
E_{L+t} \approx E_{L} \approx \frac{d^{\prime} \lambda^{\prime}\left(s^{\prime}, \bar{N}_{L}\right)}{1-\left(1-d^{\prime}\right) \lambda^{\prime}\left(s^{\prime}, \bar{N}_{L}\right) C\left(\bar{S}_{L}\right)(1-e)}
$$

for each $t>0$. Therefore we can set

$$
R_{L+1}=(1-e)^{L+1} C\left(\bar{S}_{L}\right) E_{L+1}
$$

where $(1-e)^{L+1}$ is the probability that a randomly chosen patch has age greater than $L$ and $C\left(\bar{S}_{L}\right)$ the probability that mutants survived competition and established in the first place.

Consequently, we can recursively compute, for $t=0, \ldots, L-1$,

$$
E_{L-t}=d^{\prime} \lambda^{\prime}\left(s^{\prime}, \bar{N}_{L-t}\right)+\left(1-d^{\prime}\right) \lambda^{\prime}\left(s^{\prime}, \bar{N}_{L-t}\right) C\left(\bar{S}_{L-t}\right)(1-e) E_{L-t+1}
$$

and 


$$
R_{L-t}=R_{L-t+1}+p_{L-t} C\left(\bar{S}_{L-t-1}\right) E_{L-t}
$$

until obtaining $R_{1}$, which approximates the expected number of immigrant mutant individuals produced by a mutant population during its lifetime (recall that reproductive mutants cannot establish in patches of age $\tau=0$, so that $E_{0}=0$ and $\left.R_{0}=R_{1}\right)$. The mutant fitness will then be approximated by

$$
R\left(d, s, d^{\prime}, s^{\prime}\right) \approx(1-c) R(1)
$$

\section{Appendix B. Simplified model}

To gain insight into selection on dispersal and selfing in our model, we perform a simplified analysis of the selection gradients. We do this by assuming that all the non-extinct patches have reached the stationary maximum local population $1 / b$, in other words, neglecting distribution of local population density. This simplification is thus most helpful for explaining results of the full model that do not depend on the frequency distribution of local density (see Discussion). We use the life-cycle framework developed in Massol (2013) and Massol and Débarre (2015). Our model corresponds to a juvenile dispersal life cycle characterised by the following order of events: reproduction $F^{\prime}$, dispersal $D^{\prime}$, regulation $R$, and environmental change $E$, with matrices

$$
\begin{gathered}
F^{\prime}=\left[\begin{array}{cc}
0 & 0 \\
0 & \lambda^{\prime}\left(s^{\prime}, 1 / b\right)
\end{array}\right], \\
D^{\prime}=\left[\begin{array}{cc}
\left(1-d^{\prime}\right)+d^{\prime}(1-c) e & d^{\prime}(1-c) e \\
d^{\prime}(1-c)(1-e) & \left(1-d^{\prime}\right)+d^{\prime}(1-c)(1-e)
\end{array}\right], \\
R=\left[\begin{array}{cc}
C\left(\bar{S}_{0}\right) & 0 \\
0 & C\left(\bar{S}_{1}\right)
\end{array}\right], \\
E=\left[\begin{array}{cc}
e & e \\
(1-e) & (1-e)
\end{array}\right],
\end{gathered}
$$


thus obtaining the next generation matrix as $G=E R D^{\prime} F^{\prime}$ with its detail given below:

$\left[\begin{array}{rr}0 & e\left(d^{\prime}(1-c) e \lambda^{\prime}\left(s^{\prime}, \frac{1}{b}\right) C\left(\bar{S}_{0}\right)+\left(\left(1-d^{\prime}\right) \lambda^{\prime}\left(s^{\prime}, \frac{1}{b}\right)+d^{\prime}(1-c)(1-e) \lambda^{\prime}\left(s^{\prime}, \frac{1}{b}\right)\right) C\left(\bar{S}_{1}\right)\right) \\ 0 & (1-e)\left(d^{\prime}(1-c) e \lambda^{\prime}\left(s^{\prime}, \frac{1}{b}\right) C\left(\bar{S}_{0}\right)+\left(\left(1-d^{\prime}\right) \lambda^{\prime}\left(s^{\prime}, \frac{1}{b}\right)+d^{\prime}(1-c)(1-e) \lambda^{\prime}\left(s^{\prime}, \frac{1}{b}\right)\right) C\left(\bar{S}_{1}\right)\right)\end{array}\right]$

The fitness is then the leading eigenvalue of $G$, that is

$$
\begin{aligned}
R\left(d, s, d^{\prime}, s^{\prime}\right) & \\
= & (1-e)\left(d^{\prime}(1-c) e \lambda^{\prime}\left(s^{\prime}, \frac{1}{b}\right) C\left(\bar{S}_{0}\right)\right. \\
& \left.+\left(\left(1-d^{\prime}\right) \lambda^{\prime}\left(s^{\prime}, \frac{1}{b}\right)+d^{\prime}(1-c)(1-e) \lambda^{\prime}\left(s^{\prime}, \frac{1}{b}\right)\right) C\left(\bar{S}_{1}\right)\right)
\end{aligned}
$$

The sign of the selection gradient on selfing

$$
\left.\frac{\partial}{\partial s^{\prime}} R\left(d, s, d^{\prime}, s^{\prime}\right)\right|_{\left(d^{\prime}, s^{\prime}\right)=(d, s)}
$$

turns out to simply be given by the sign of

$$
\frac{\partial}{\partial s^{\prime}} \lambda^{\prime}\left(s^{\prime}, \frac{1}{b}\right)=f\left((1-\delta)-\frac{1}{2(1+a b)}\right)
$$

that is, $s_{E S S}=0$ if $(1-\delta)<1 /(2(1+a b))$ or $s_{E S S}=1$ otherwise. Notably, selection on selfing does not depend on the traits $(d, s)$ themselves, supporting our observation of selection for extreme mating strategies (either full selfing or full outcrossing) in our model. Instead, the ESS of dispersal rate is the one that annihilates the selection gradient 


$$
\left.\frac{\partial}{\partial d^{\prime}} R\left(d, s, d^{\prime}, s^{\prime}\right)\right|_{\left(d^{\prime}, s^{\prime}\right)=(d, s)}=(1-e)\left((1-c) e \lambda\left(s, \frac{1}{b}\right) C\left(\bar{S}_{0}\right)+\left(-\lambda\left(s, \frac{1}{b}\right)+\right.\right.
$$

$$
\left.\left.(1-c)(1-e) \lambda\left(s, \frac{1}{b}\right)\right) C\left(\bar{S}_{1}\right)\right)
$$

which can be found by substituting the expressions of $C\left(\bar{S}_{0}\right)$ and $C\left(\bar{S}_{1}\right)$ with

$$
\bar{S}_{0}=\frac{d(1-c)(1-e) \lambda\left(s, \frac{1}{b}\right)}{b}
$$

and

$$
\bar{S}_{1}=\frac{(1-d) \lambda\left(s, \frac{1}{b}\right)}{b}+\frac{d(1-c)(1-e) \lambda\left(s, \frac{1}{b}\right)}{b} .
$$

The result is

$$
\left.d_{E S S}\right|_{S_{E S S}=0}=\frac{e}{e+c(1-e)}-\frac{c(1+a b)}{f(1-c)(e+c(1-e))}
$$

and

$$
\left.d_{E S S}\right|_{S_{E S S}=1}=\frac{e}{e+c(1-e)}-\frac{c}{f(1-\delta)(1-c)(e+c(1-e))} .
$$

Notice that when $f$ is large (assumption of the classic Wright (1931) island model) the ESS of dispersal rate converges to the known result of $d_{E S S}=e /(e+c(1-e))$ (Massol and Débarre 2015). Notice that, as long as $(1-\delta) \leq 1 /(1+a b)$, the ESS dispersal rate $d_{E S S}$ with full selfing $\left(S_{E S S}=1\right)$ is lower than the ESS dispersal rate with full out-crossing $\left(s_{E S S}=0\right)$, consistent with the negative effect of selfing on dispersal presented in the Results and Discussion. This negative effect is maximal when $(1-\delta)=1 / 2(1+a b)$ (i.e. when $S_{E S S}$ transitions from full out-crossing to full selfing). Therefore, the automatic transmission advantage of selfing is responsible for this negative effect on dispersal. Moreover, the effect 
of each parameter can be computed. As for selection on selfing, $\delta$ (respectively, $a b$ ) have a negative (respectively, positive) effect. If the selection gradient is close to singular ((1$\delta)=1 / 2(1+a b))$, then a small parameter perturbation can trigger the transition of $s_{E S S}$ from 0 to 1 or vice versa. The sign of the effect of each parameter on dispersal is given in supplementary tables (Tables S2-S4). Such results are in good qualitative agreement with those presented in the main text (especially see Figure 3). 